Supporting Information for

\title{
3D-Printed Carbon Nanoelectrodes for In Vivo
}

\section{Neurotransmitter Sensing}

Qun Cao, ${ }^{1}$ Mimi Shin, ${ }^{1}$ Nickolay V. Lavrik, ${ }^{2}$ and B. Jill Venton ${ }^{1, *}$

1Department of Chemistry, University of Virginia, Charlottesville, Virginia 22904, United States

${ }^{2}$ Center for Nanophase Material Science, Oak Ridge National Lab, Oak Ridge, Tennessee 37831, United States

Email: jventon@virginia.edu 


\section{Methods}

\section{Chemicals and Materials}

Dopamine and hexaammineruthenium (III) chloride were purchased from Sigma-Aldrich (St. Louis, MO). Phosphate-buffered saline (PBS) buffer was prepared with $131.5 \mathrm{mM} \mathrm{NaCl}, 3.25$ $\mathrm{mM} \mathrm{KCl}, 1.2 \mathrm{mM} \mathrm{CaCl}_{2}, 1.25 \mathrm{mM} \mathrm{NaH}_{2} \mathrm{PO}_{4}, 1.2 \mathrm{mM} \mathrm{MgCl}_{2}$, and $2.0 \mathrm{mM} \mathrm{Na}_{2} \mathrm{SO}_{4}$, and the $\mathrm{pH}$ was adjusted to 7.4 by concentrated $\mathrm{NaOH}$. $10 \mathrm{mM}$ stock solution of dopamine was prepared in $0.1 \mathrm{M}$ perchloric acid and diluted to $10 \mu \mathrm{M}$ with PBS. In cyclic voltammetry experiments, $10 \mathrm{mM}$ hexaammineruthenium(III) chloride solution was prepared in PBS buffer. All aqueous solutions were made with deionized water (EMD Millipore, Billerica, MA). The niobium wires with $50 \mu \mathrm{m}$ were purchased from Advent Research Materials Ltd (Oxford, England).

\section{D-Printing of the Nanoelectrodes}

3D structures were designed and drawn in SolidWorks (Dassault Systèmes SolidWorks Corporation, MA) and imported to Nanoscribe Pro GT laser lithography system. CAD model of the printed structures are shown in Figure S1.

a)

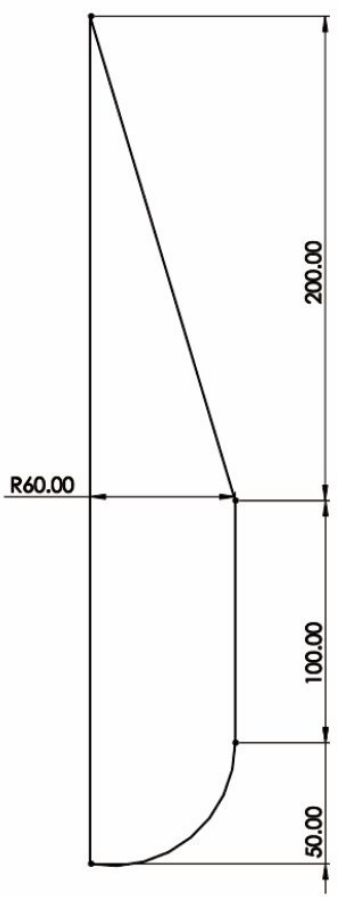

b)

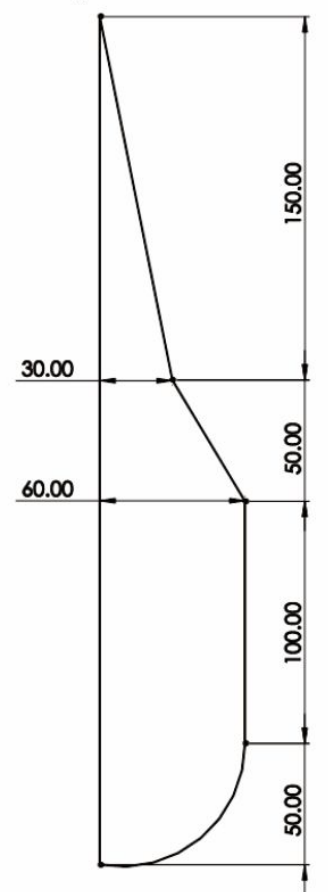

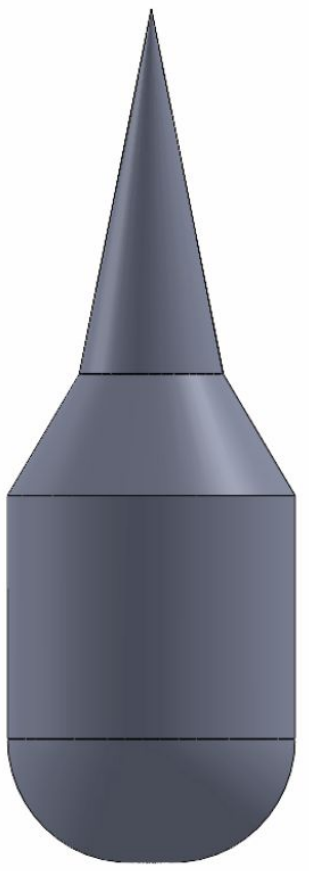

Figure S1. CAD model of the printed structures (unit $\mu \mathrm{m}$ ). a) Geometry 1 and b) Geometry 2.

Prior to direct laser writing, the $50 \mu \mathrm{m}$ niobium anchor wires were etched to several microns around the tip. Thin metal wire can enhance the mechanical stability, and diminish the shadow created by the wires. An AC current supplier (Interworld Highway LLC, Long Branch, NJ) was used to etch the niobium wires. The wires were cut to approximately $2 \mathrm{~cm}$ in length, and etched in batch at $2 \mathrm{~V}$ and $60 \mathrm{~Hz}$ in concentrated $\mathrm{HCl}$ for 20 min. 
Nanoscribe Pro GT laser lithography system (Nanoscribe $\mathrm{GmbH}$, Karlsruhe, Germany) were used for 3D direct-write fabrication. The system was equipped with a femtosecond pulsed (100 fs, $50 \mathrm{~mW}$ ) fiber laser (FemtoFiber Pro, Toptica Photonics) at $780 \mathrm{~nm}$ and an oil-immersion objective $(25 X, N . A .=0.8)$. To set up the stage, etched niobium wires were immobilized horizontally on a silicon wafers, providing an anchor point and electric connection for the 3Dprinted structures. A tape is put underneath the wires to create a gap so that the 3D-printed structures don't attach to the silicon wafer. Niobium was used as the metal substrates because its lack of electroactivity for neurotransmitters, the outstanding electrochemical inertness, and the relatively low-cost compared to palladium and tantalum. ${ }^{1} \mathrm{~A}$ layer of the negative photopolymer, IP-S (Nanoscribe $\mathrm{GmbH}$, Karlsruhe, Germany) was added to fully cover the anchored metal wires. About 15 niobium wires can be mounted on the wafer in one batch. The 3D structures were printed using Adaptive slicing mode and Shell \& Scaffold fill mode. The slicing distance was $0.6 \mu \mathrm{m}$ and the hatching distance was $0.4 \mu \mathrm{m}$. The hatching angle was 90 degree. The rest of the parameters were as default. With these parameters, the printing time for each structure is approximately $6 \mathrm{~min}$. After the laser 3D-printing, the samples were developed in SU-8 developer for $15 \mathrm{~min}$, to remove the volume not polymerized.

\section{Pyrolysis}

The pyrolysis process was performed by First Nano Rapid Thermal Processor (RTP) (Firstnano CVD Equipment Corporation, NY), which uses an infrared lamp heating system to control the rapid thermal ramps. The pyrolysis of polymerized IP-S included two steps: $450{ }^{\circ} \mathrm{C}$ in oxygen $(101 \mathrm{kPa})$ and $\operatorname{argon}(101 \mathrm{kPa})$ at $900{ }^{\circ} \mathrm{C}, 10 \mathrm{~min}$ for each step. The shrinkage factor $\mathrm{S}$ was calculated using the equation $S=1-\frac{\text { Parameter of pyrolysed carbon }}{\text { Parameter of polymer }}$. The length and width of the structure were measured under scanning electron microscope.

\section{SEM, FIB and EDS}

Scanning electron microscope (SEM) images were taken by Phenom XL SEM (Nanoscience Instruments, Phoenix, AZ) using secondary electron detector at an accelerating voltage of $10 \mathrm{kV}$. Focused ion beam (FIB) milling was performed by Helios UC G4 Dual Beam FIB-SEM (Thermo Fisher Scientific, Waltham, MA) site-specific modifications of samples using the Ga ion-beam unit. The electron detector for FIB is Ion Conversion and Electron (ICE) detector at an accelerating voltage of $30 \mathrm{kV}$. Another SEM detector, Everhart-Thornley detector (ETD), was aligned at 52 degrees with FIB detector. Both detectors were used to acquire SEM images. To polish the nanotip into disk-shape, a reference line was drawn under SEM to mill the nanotip into $600 \mathrm{~nm}$ disk. A current of $0.79 \mathrm{nA}$ was applied for FIB milling, and for each sample this process takes 10 second to 1 minute. Energy Dispersive X-ray Spectroscopy (EDS) was done using the same instrument. An accelerating voltage of $5 \mathrm{kV}$ and current of $1.6 \mathrm{nA}$ was used to map the element distribution of aluminum, carbon and oxygen.

\section{Raman Spectroscopy}

Raman spectroscopy measurements were performed with a Renishaw 100 confocal microRaman system (Renishaw, Hoffman Estates, IL) with 1800 lines/mm diffraction grating and 632 $\mathrm{nm} \mathrm{He}-\mathrm{Ne}$ laser. Laser intensity was $10 \%$ and scan range was $100 \mathrm{~cm}^{-1}$ to $3200 \mathrm{~cm}^{-1}$. 
Atomic Layer Deposition

Atomic Layer Deposition was performed on FlexAl Atomic Layer Deposition (Oxford Instruments, Concord, MA. To deposit a $100 \mathrm{~nm}$ layer of aluminum oxide, trimethylaluminium (TMA) precursor was used and 741 cycles of plasma ALD was performed (each cycle $1.35 \AA$ ) at a temperature of $150^{\circ} \mathrm{C}$.

\section{Parylene Coating}

Parylene- $\mathrm{N}$ was deposited on the carbonized structure using PDS 2010 parylene coating system (Specialty Coating Systems Inc., Indianapolis, IN). $0.7 \mathrm{~g}$ of parylene dimer precursor was used, and the coating thickness was about $130 \mathrm{~nm}$. Vaporizer set point was $160{ }^{\circ} \mathrm{C}$ and furnace set point was $650^{\circ} \mathrm{C}$.

\section{Cyclic Voltammetry}

Cyclic voltammetry experiments at slow scan rates were performed with Reference 600 potentialstat (Gamry Instruments, Warminster, PA). The ends of metal wires without carbon microstructures were connected to silver wires to achieve the connection. Three-electrode system was used, the 3D-printed electrode was the working electrode, the reference electrode was a standard $\mathrm{Ag} / \mathrm{AgCl}$ electrode, and $\mathrm{Pt}$ wire was used as the counter electrode. A waveform scanned from $-0.4 \mathrm{~V}$ to $0.1 \mathrm{~V}$ was used for hexaammineruthenium(III) at a scan rate of 100 $\mathrm{mV} / \mathrm{s}$.

\section{Fast-scan Cyclic Voltammetry}

FSCV experiments were performed with ChemClamp potentiostat and headstage (Dagan, Minneapolis, MN). A triangular waveform was used. Holding potential was $-0.4 \mathrm{~V}$, switching potential was $+1.3 \mathrm{~V}$, scan rate was $400 \mathrm{~V} / \mathrm{s}$, and repetition frequency is $10 \mathrm{~Hz}$. Two-electrode system was applied using working electrode versus $\mathrm{Ag} / \mathrm{AgCl}$ reference electrode. The PBS buffer and dopamine solutions were injected through the flow cell at $2 \mathrm{~mL} / \mathrm{min}$ by a syringe pump (HarvardApparatus, Holliston, MA) and a flow-injection system consisting of a six-port loop injector with an air actuator (VIVI Valco Instruments, Houston, TX). The data were collected with HDCV Analysis software (Department of Chemistry, University of North Carolina at Chapel Hill).

\section{Fruit Fly Experiments}

Dopamine transporter (DAT)-Gal4 and UAS-GFP were obtained from the Bloomington Stock Center (Indiana University, Bloomington, IN) and crossed to generate a fly expressing GFP in dopaminergic neurons. 4 to 10 day old adult Drosophila was anesthetized by rapid chilling procedure; an empty Petri dish was placed in ice, and then flies were put on the chilled Petri dish for $5 \mathrm{~min}$. The anesthetized fly was placed in a chilled phosphate buffer (PBS) dissecting buffer $\left(4^{\circ} \mathrm{C}\right)$ with $11.1 \mathrm{mM}$ glucose and $5.3 \mathrm{mM}$ trehalose. Then, the fly was decapitated and the brain was isolated under a dissecting stereoscope. The harvested brain was transferred to a Petri dish containing room-temperature dissecting PBS. Brains were situated dorsal side up to 
provide easy access for the electrode and a pulled glass pipette containing either $5 \mathrm{mM}$ acetylcholine. All the experiments were conducted under a SMZ800N stereoscope (Nikon Instruments Inc., NY), and brains were allowed to equilibrate $15 \mathrm{~min}$ prior to the experiment. The electrode and pipette were placed using a micromanipulator (Narishige Inc., Amityville, NY). The electrode was positioned the corner of the mushroom body and pipette was placed approximately 10 to $15 \mu \mathrm{m}$ away from the tip of the electrode at the same depth. A Picospriter III (parker Hannfin, Fairfield, NJ) was used to precisely pressure eject acetylcholine or dopamine into the isolated brain. Prior to the experiment, a pipette was calibrated by picospritzing a droplet in oil and measuring the diameter of ejected droplet using DS-Qi2 monochrome CMOS camera and NIS-Elements BR imaging software (Nikon Instruments Inc. Melville, NY). The amount of acetylcholine and dopamine ejected was controlled by varying ejected volumes, which relies on different pulse durations. 


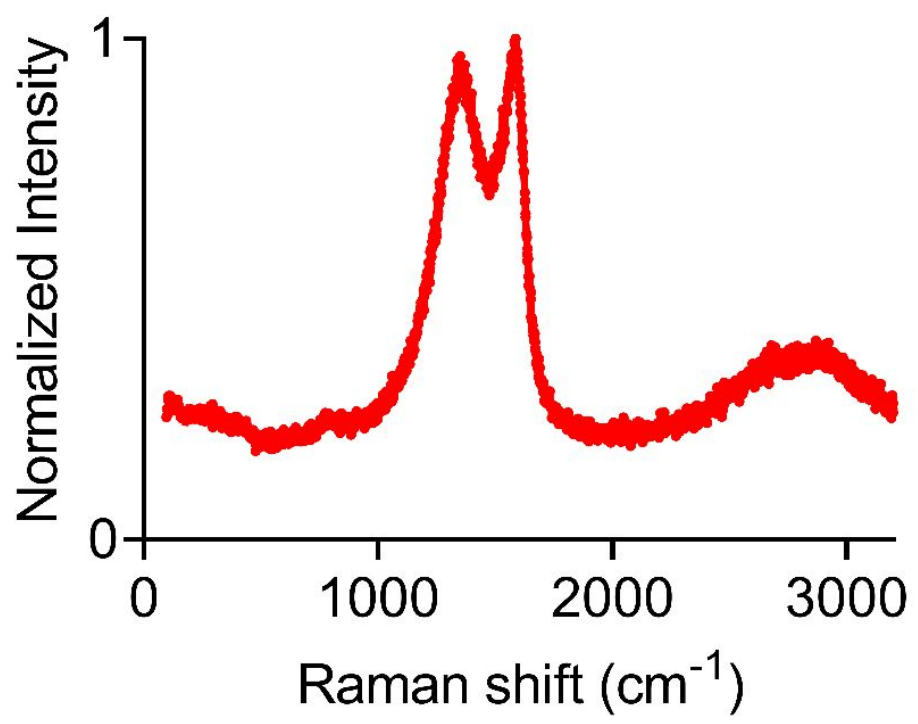

Figure S2. Raman spectra of the carbonized structure. 
Table S1. Size and shrinkage measurements of the base of the electrodes.

\begin{tabular}{|l|c|c|c|c|c|c|c|c|}
\hline & \multicolumn{4}{|c|}{ Width $(\mu \mathrm{m})$} & \multicolumn{4}{c|}{ Length $(\mu \mathrm{m})$} \\
\hline & Designed & Printed & Pyrolyzed & Shrinkage & Designed & Printed & Pyrolyzed & Shrinkage \\
\hline Geometry1 & 120 & $115 \pm 2$ & $49 \pm 2$ & $57 \%$ & 350 & $350 \pm 4$ & $128 \pm 5$ & $63 \%$ \\
\hline Geometry2 & 120 & $118 \pm 9$ & $48 \pm 5$ & $59 \%$ & 350 & $351 \pm 9$ & $122 \pm 9$ & $68 \%$ \\
\hline
\end{tabular}

Values are mean \pm standard deviation, $n=5$. 


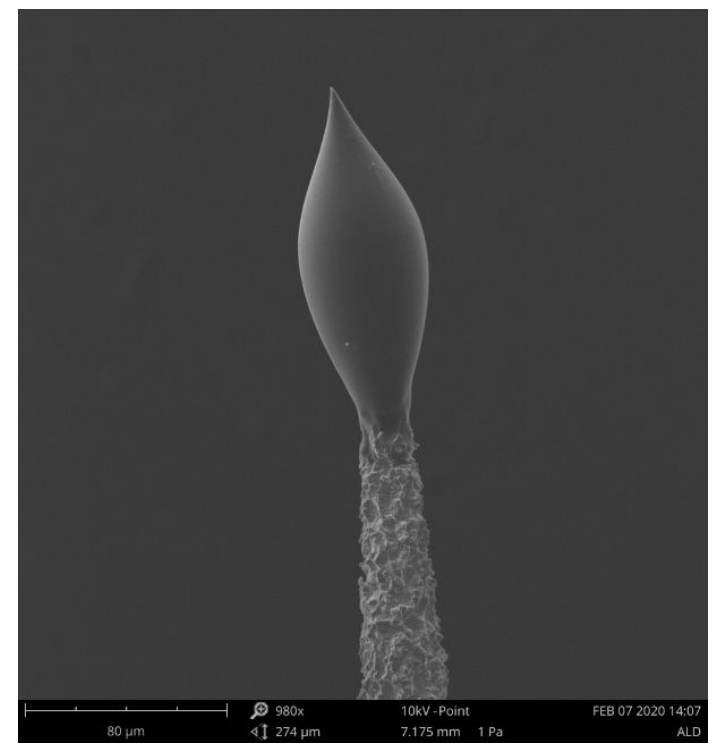

Figure S3. Carbonized structure coated with an insulation layer of $\mathrm{Al}_{2} \mathrm{O}_{3}$ by atomic layer deposition. The thickness of the coating layer is $100 \mathrm{~nm}$. 

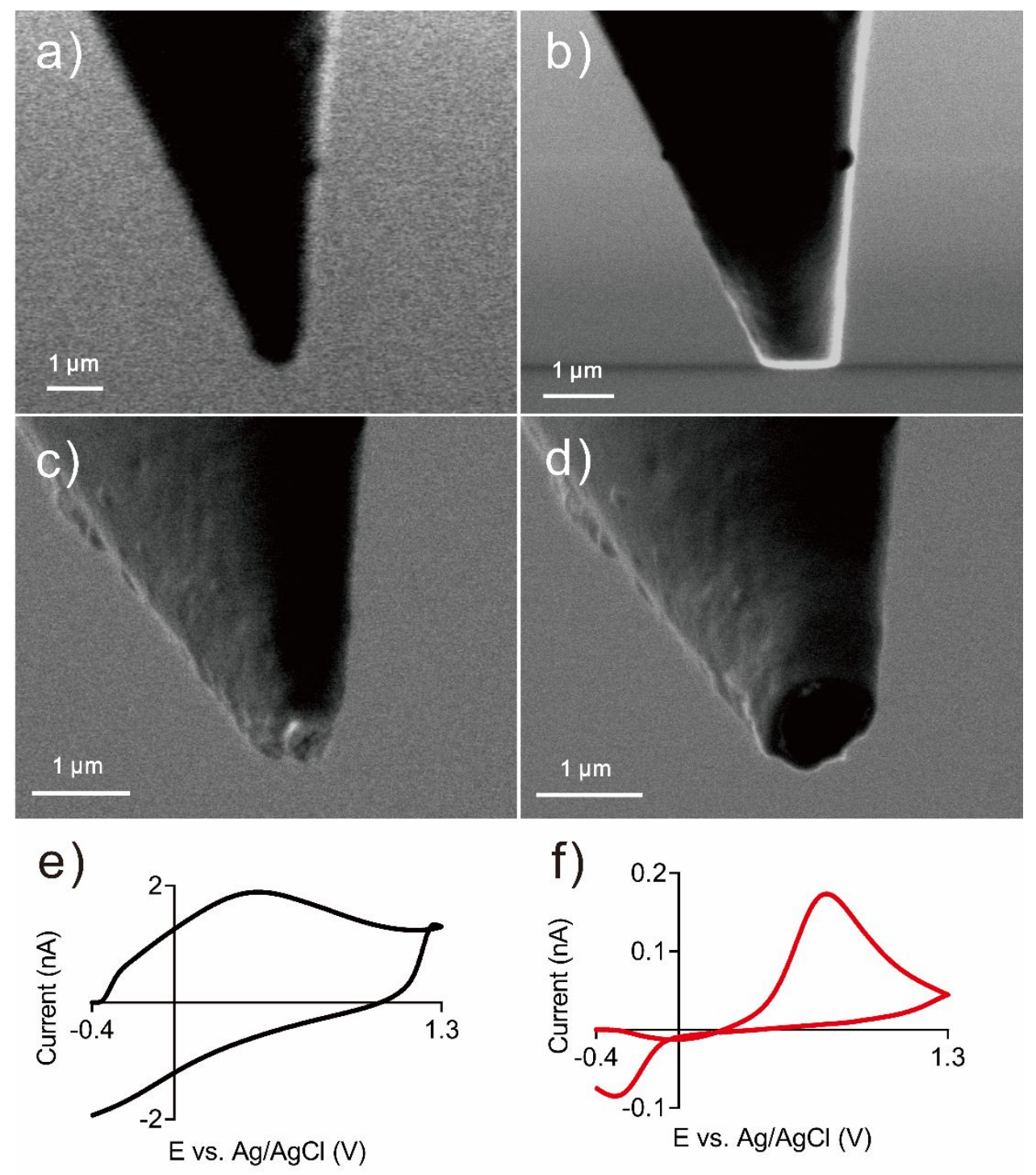

Figure S4. Parylene coating and FIB milling. FIB detector view: a) before FIB polish, b) after FIB polish; SEM detector view: c) before FIB polish, d) after FIB polish. FSCV study of 3D-printed nanoelectrodes. e) Background current. f) Background-subtracted current of $10 \mu \mathrm{M}$ dopamine. 

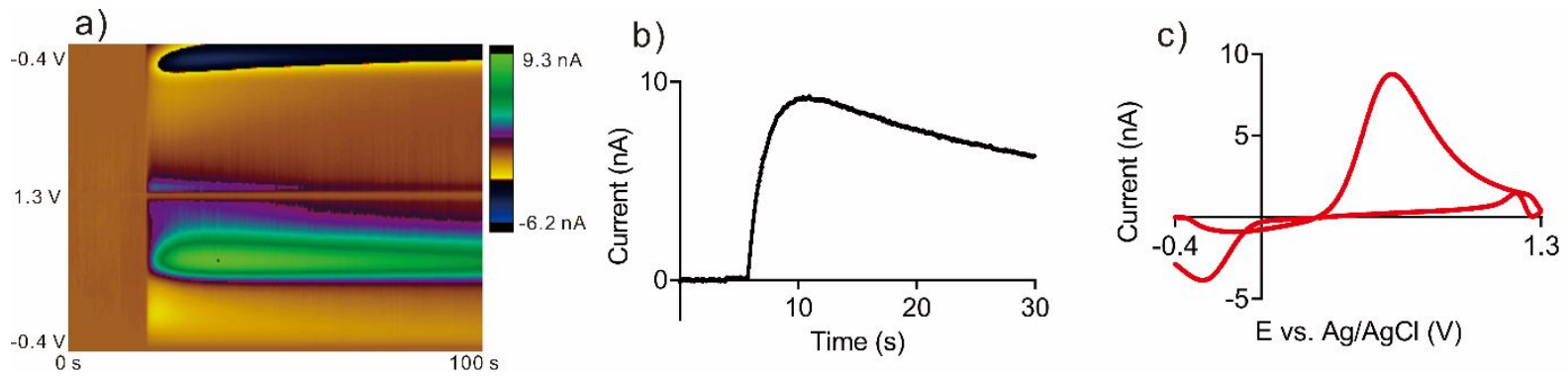

Figure S5. Detection of exogenous dopamine in adult fruit fly brain. At 5s, $1 \mathrm{mM}$ dopamine was applied exogenously. a) False color plot. b) Puffed on dopamine current versus time. c) Cyclic voltammogram of the exogenous dopamine. 


\section{$\underline{\text { References }}$}

(1) Zestos, A. G.; Yang, C.; Jacobs, C. B.; Hensley, D.; Venton, B. J. Carbon Nanospikes Grown on Metal Wires as Microelectrode Sensors for Dopamine. Analyst 2015, 140 (21), 7283-7292. 